\title{
Editorial
}

\section{Offering choices}

The idea that people should be enabled to make informed choices about their own health care is a key theme in much contemporary thinking about participation in health care, but two papers in this issue of Health Expectations remind us that the offering of choice does not always attract universal support.

Chris Woodrow et al. ${ }^{1}$ convened focus groups of people aged between 60 and 69 years to consider drafts of an information leaflet that would accompany a mailed invitation to participate in a national bowel cancer screening programme. The leaflet was intended to promote informed choice about participation, and included information about the negative as well as the positive aspects of screening and its possible consequences. Some focus group participants considered this inappropriate. They thought that the negative aspects of screening should be downplayed and that participation in the screening programme should be strongly promoted rather than presented as a matter of choice. $^{1}$

Kimberley Chiew et al. ${ }^{2}$ invited medical oncologists and women with metastatic breast cancer to review a decision aid that presented information about two main healthcare options for people with hormone-resistant metastatic breast cancer: supportive care with chemotherapy and supportive care without chemotherapy. Two of the seven oncologists who agreed to review the decision aid did not think it would be useful because they regarded supportive care without chemotherapy as a less desirable option. $^{2}$
Differences of opinion about whether and how particular healthcare interventions should be offered are not surprising: people have diverse experiences and values relating to health, healthcare technologies, and the respective roles of patients and health professionals. However, the question of how different opinions should be reflected in communication about healthcare options remains difficult.

The developers of information resources who aspire to consider the views of potential users as they shape the content and presentation of their resources need to exercise careful judgement as they attempt to synthesize and adjudicate between diverse views. Those who are committed to policies of promoting informed choice might, of course, choose to ignore or limit the influence of people who in some sense oppose this. Although the robustness of their justifications for this might warrant further examination, the approach does tend to preserve the scope for those who want to make informed choices to do so.

When health professionals oppose the offering of choice, however, a broader set of issues are raised. Information developers who refuse to allow those professionals' views to impact on the content and tone of their resources will probably struggle to get those resources to their intended audience: as Silva et $a .^{3}$ report in this issue, a lack of physician support can be an important barrier to the use of decision aids. Potentially more seriously, health professionals who oppose the offering of particular choices (or choice more generally) might unduly limit the options 
available to those patients who consult with them, precluding discussion of and access to interventions that many of their professional colleagues (and informed patients) would regard as reasonable.

In the case of the medical oncologists who are disinclined to regard supportive care without chemotherapy as something worth telling women with metastatic breast disease about, concern might be mitigated by the thought that patients usually have a right to decline treatment. However, if not having chemotherapy is not explicitly mentioned as an option (not even as one that the doctor would not recommend), patients who might prefer to decline chemotherapy are less likely to feel they have scope to explore this possibility, or to feel confident that they will continue to be respected and supported if they assert themselves and say 'No, thank you'. Patients are unlikely to be able to avoid the problem by steering clear of health professionals who offer a more limited range of options than their colleagues because they may not be aware of the range of options that are considered reasonable by a significant proportion of members of a profession and often cannot tell which of those members would be willing to discuss a broader rather than a narrower option set.

Variations of opinion within health professional groups are often not well characterized or documented, so it is hard to ascertain their significance. However, the questions of whether, when and how health professionals' personal opinions about particular interventions can justify them not mentioning them to patients have not been well explored. The Good Medical Practice guidance developed in the UK requires doctors to keep their professional knowledge and skills up to date, to recognize and work within the limits of their competence, to work with colleagues in ways that serve patients' interests, and to respect patients' rights to reach decisions with them about their treatment and care. ${ }^{4}$ What it says in relation to these requirements, however, does not amount to clear guidance about doctors' responsibilities to recognize when their own views about what might be acceptable include a narrower range of options than most of their colleagues, and it does not stipulate whether and how they should act to ensure patients are not adversely affected by this. This perhaps reflects a broader lack of debate about which constraints on choice should be disclosed to patients and how. ${ }^{5}$ Health Expectations will welcome contributions that further that debate.

\section{Vikki Entwistle}

Editor

Health Expectations, Social Dimensions of Health Institute, Universities of Dundee and St Andrews

\section{References}

1 Woodrow C, Watson E, Rozmovits L, Parker R, Austoker J. Public perceptions of communicating information about bowel cancer screening. Health Expectations, 2008; 11: 18-27.

2 Chiew KS, Shepherd H, Vardy J, Tattersall MHN, Butow PN, Leighl NB. Development and evaluation of a decision aid for patients considering first-line chemotherapy for metastatic breast cancer. Health Expectations, 2008; 11: 37-47.

3 Silva KA, Ozanne EM, Sepucha KR. Implementing breast cancer decision aids in community sites: barriers and resources. Health Expectations, 2008; 11: 48-55.

4 General Medical Council. Good Medical Practice: Guidance for Doctors. London: General Medical Council, 2006.

5 Entwistle V, Williams B, Skea Z, Maclennan G, Bhattacharya S. Which surgical decisions should patients participate in and how? Reflections on women's recollections of discussions about variants of hysterectomy. Social Science and Medicine, 2006; 62: 499-509. 